\title{
Diagnosis of Pathologically Early HCC with EOB-MRI: Experiences and Current Consensus
}

\author{
Tomoaki Ichikawa Katsuhiro Sano Hiroyuki Morisaka \\ Department of Radiology, University of Yamanashi, Yamanashi, Japan
}

\section{Key Words}

Early hepatocellular carcinoma $\cdot$ Gadoxetic acid $\cdot$ MRI $\cdot$ OATP1B3

\begin{abstract}
Objective: After much debate, the International Consensus Group for Hepatocellular Neoplasia (ICGHN) has recently arrived at a conclusion regarding the pathological criteria for early hepatocellular carcinoma (HCC). They have stated that stromal invasion should be recognized as the most important pathological finding for precisely diagnosing and differentiating early HCC from dysplastic nodules (DN). Methods: We conducted a review of the imaging findings from multi-imaging modalities of early HCC cases diagnosed according to the pathological criteria of the ICGHN. The multi-imaging modalities included gadoxetic acid (Gd-EOB-DTPA) enhanced magnetic resonance imaging (MRI), abbreviated as EOB-MRI, contrast-enhanced $\mathrm{CT}$ (CE-CT), CT during arterioportography (CTAP), and CT during hepatic arteriography (CTHA). It has been shown that EOB-MRI is the only imaging modality that has sufficient resolution for the detection and classification of early HCC. Results: The most significant imaging feature for diagnosing early HCC was hypointensity on hepatobiliary-phase (HP) images of EOB-MRI; all of the cases of early HCC that were detected on HP images of EOB-MRI showed hypointensity, while all of the images of DN showed isointensity or hyperintensity compared with the liver parenchyma. The results of the diagnostic performance analysis showed that EOB-MRI had excellent sensitivity (97\%) for detecting early HCC and outstanding specificity (100\%) for distinguishing early HCC from DN.

Conclusions: Considering the results from imaging-pathologic correlations and follow-up studies indicating that many early-stage hepatocellular nodules showing hypointensity on HP images of EOB-MRI tend to develop hypervascularization during a relatively short followup period, it is beginning to be accepted that such nodules may be treated as early HCC.
\end{abstract}


However, hepatologists and radiologists should also recognize that some cases of early HCC may show isointensity or hyperintensity on HP images of EOB-MRI, making it impossible to differentiate early HCC from DN, although the low prevalence of such nodules may be unlikely to affect the generally accepted follow-up protocols for cirrhotic patients.

Our results and other recent reports have indicated that signal-intensity patterns on HP images of EOB-MRI for DN and early HCC directly correlate with the degree of expression of the organic anion transporting polypeptide (OATP) 1 B3 in the nodules. Thus, the diagnostic performance of pathological analyses for early HCC cases may be dramatically improved, nearly up to that of EOB-MRI, by incorporating an OATP1B3 staining method.

Copyright @ 2014 S. Karger AG, Basel

\section{Introduction}

It has been recognized that following treatment, early hepatocellular carcinoma (HCC) shows a longer time-to-recurrence and a higher 5-year survival rate compared to those in which it has progressed. Therefore, the detection of tumors at an early stage is important to potentially decrease the mortality of this disease [1].

HCC has been shown to develop by multistep carcinogenesis from a low-grade dysplastic nodule (DN) to a high-grade DN followed by an early HCC and finally, a progressed HCC [2, $3]$. However, the precise detection of an early HCC and the accurate differentiation between an early HCC and a DN have been difficult and remain unclear to date. The main cause of these issues is that the pathological features of an early HCC are very close to those of a DN [4]. As a result, the distinct pathological definition of an early HCC was not established until recently, despite much debate among renowned hepatopathologists [5, 6].

In 2009, the International Consensus Group for Hepatocellular Neoplasia (ICGHN), which was composed of 34 world-renowned pathologists and two clinicians, coming from a total of 13 countries, and which was based on the framework of the previous International Working Party (IWP) [5], finally announced a consensus on the pathological criteria of early HCC after significant debate [7]. As a special mention in this report, stromal invasion was recognized as the most important objective pathological finding for differentiating an early HCC from a DN.

Previous reports that tried to assess the diagnostic performance of various imaging modalities for evaluating early HCC, prior to the establishment of the ICGHN criteria, often lacked reliability and reproducibility owing to the vague and subjective pathological standards of reference. For this reason, the diagnostic abilities of each imaging modality for the detection of early HCC and the differentiation between an early HCC and a DN were re-evaluated and clarified based on the precise pathological diagnosis of early HCC using the ICGHN criteria [8]. Among the various imaging modalities, magnetic resonance imaging (MRI) with gadoxetic acid (Gd-EOB-DTPA [Primovist]; Bayer Schering Pharma, Berlin, Germany) (EOBMRI) has taken on an increasingly important role, and which became available at the same time as the landmark announcement by the ICGHN [9]. EOB-MRI allows clinicians to assess tumor vascularity on hepatic arterial-dominant phase (HAP) images and enables the acquisition of hepatocyte-phase (HP) images with the uptake of approximately $50 \%$ of the GdEOB-DTPA by the hepatocytes $[10,11]$. Early reports describing the results of multicenter phase III studies [12-15] predicted that early HCC might show hypointensity on HP images, similar to the majority of progressed HCC, because of the lack of uptake of Gd-EOB-DTPA [15]. Therefore, EOB-MRI can provide excellent lesion detection of early (hypovascular) HCC by HP images. Moreover, the ability of EOB-MRI may possibly extend to the accurate differ- 

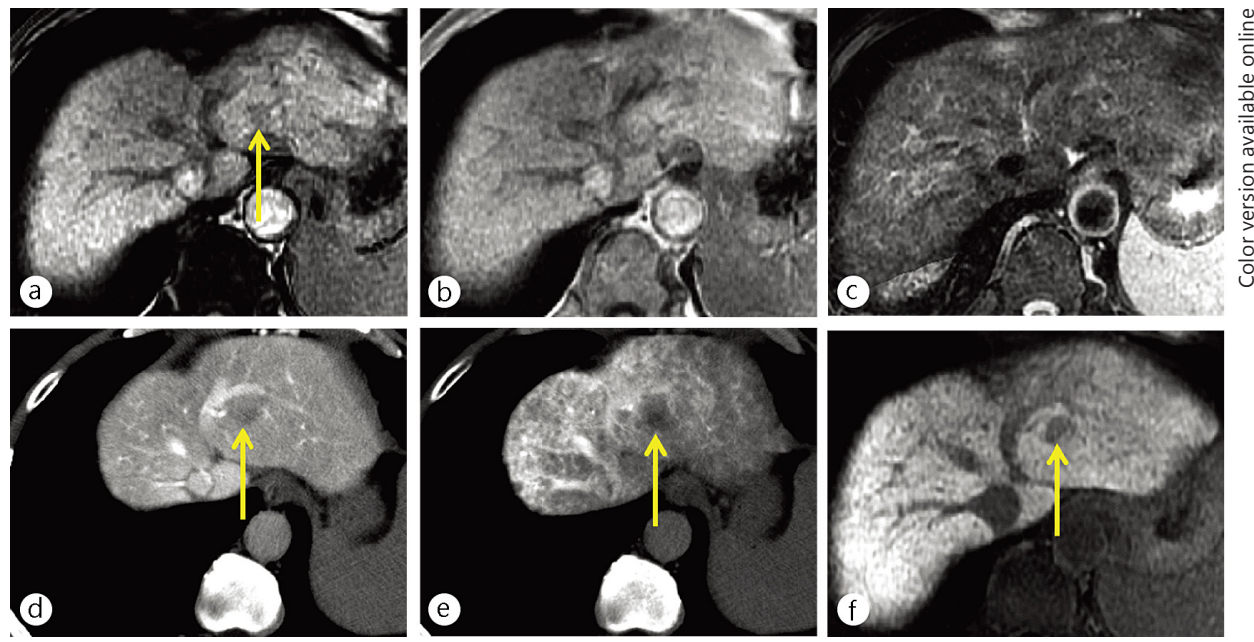

Fig. 1. A 65-year-old man with early HCC (yellow arrows). The nodule was demonstrated as a hypointensity on opposed-phase T1-weighted MR imaging (a). There was no nodule on in-phase T1-weighted (b) and T2-weighted (c) MR images. The nodule was identified as slight hypoattenuation on CT during arterioportography (d) because the nodule showed slightly decreased portal flow compared with surrounding liver parenchyma. The nodule also showed slightly decreased arterial flow on CT during hepatic arteriography (e). The nodule was clearly demonstrated as exhibiting hypointensity on hepatocyte imaging of EOB-MRI (f).

entiation between an early HCC and a DN with HP images, which has not been achieved by any previous imaging techniques [8].

In this review article, we will outline the current understanding and a recent consensus of the diagnostic ability of several imaging modalities for the diagnosis of early HCC. This will focus on the ability of EOB-MRI to detect early HCC, and to differentiate between an early HCC and a DN, using both our research data on surgically resected hepatocellular nodules (based on the strict pathological diagnoses by the ICGHN criteria), as well as recently published reports.

\section{Detection and Differentiation of Early HCC with EOB-MRI}

\section{Clinical Significance of Hypointensity on HP Images of EOB-MRI}

In undertaking a study to ascertain the most significant imaging findings for diagnosing early HCC, the use of definite pathological diagnoses, in accordance with the ICGHN criteria, was paramount [7]. Therefore, we included only surgically resected nodules so that the pathologists could thoroughly investigate whole nodules. Moreover, the diagnosis for each hepatocellular nodule included in our investigation was made in strict accordance to the ICGHN pathological criteria by a blinded hepatopathologist, who was a member of the ICGHN. Another ICGHN hepatopathologist, diagnosed the lesions independently to evaluate inter-observer variability. If there was disagreement in the diagnoses for any nodules, the final diagnosis was made by consensus [8].

In our study, the most important imaging feature of EOB-MRI proved to be hypointensity on HP images for both detecting early HCC and differentiating between an early HCC and a DN. This was superior when compared to other imaging modalities, such as contrast-enhanced CT (CE-CT), unenhanced MRI, CT during arterioportgraphy (CTAP), and CT during hepatic arteriography (CTHA) (fig 1-3). Using the finding of hypointensity on HP images, the sensitivity of 

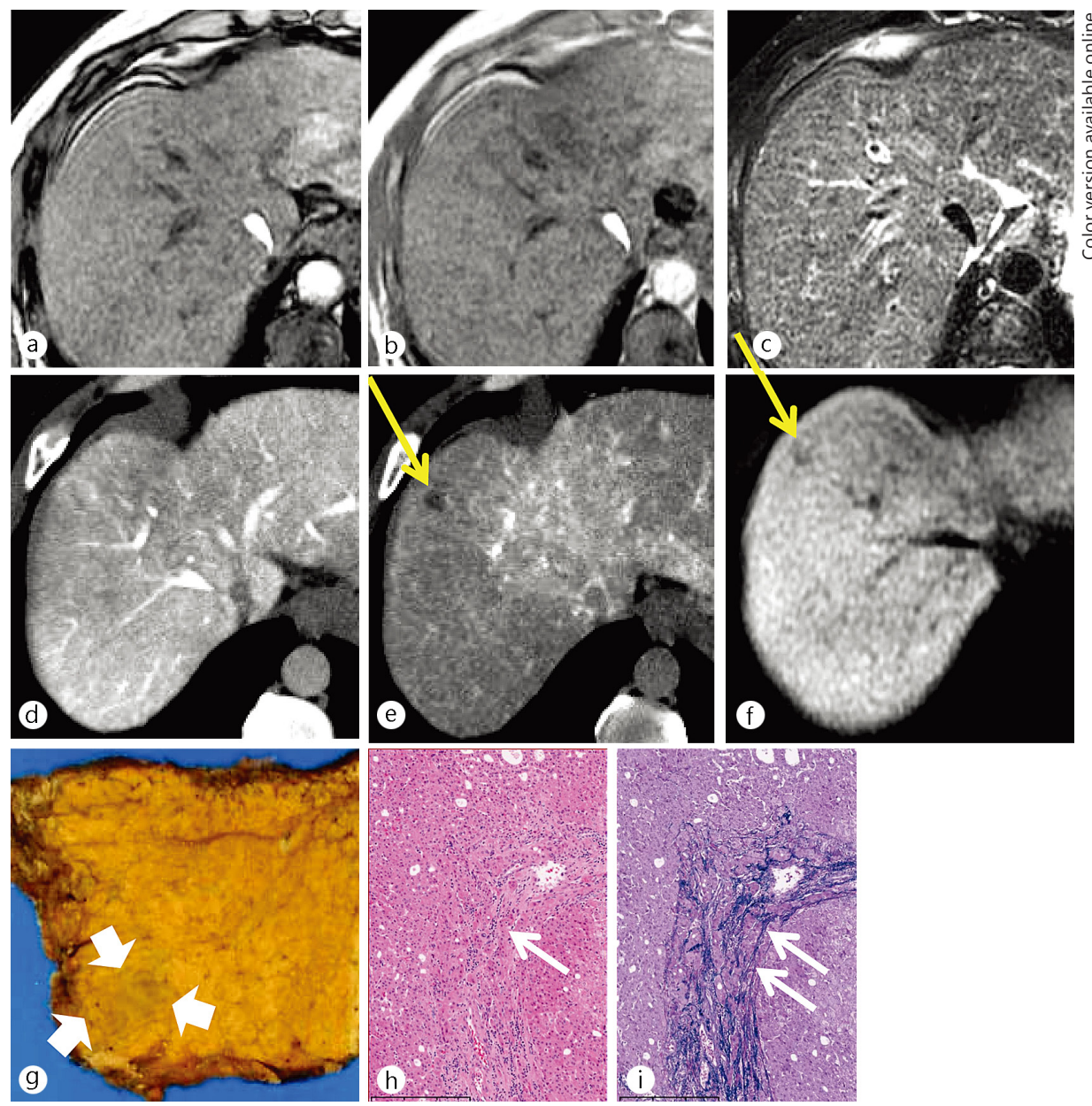

Fig. 2. A 50-year-old man with an early HCC. There was no nodule on the opposed-phase (a), the inphase (b) T1-weighted MR images or the T2-weighted MR image (c). There was no nodule on CT image during arterioportography (d) because the nodule showed equivalent portal flow compared with the surrounding liver parenchyma. The nodule (yellow arrows) showed decreased arterial flow on CT imaging during hepatic arteriography (e). The nodule was detected as hypointense on hepatocyte imaging via EOB-MRI (f). On histological examination, this nodule was identified as a small nodule with an unclear margin (thick white arrows) macroscopically (g). Portal invasion (white short arrows), which is a unique finding to early HCC, was identified on hematoxylin and eosin staining (h) and Victoria-blue staining (i), respectively.

detecting early HCC with EOB-MRI was excellent (97\%), which was far more sensitive than other imaging modalities (range, 57-73\%). Similarly, an excellent specificity (100\%) for differentiating between an early HCC and a DN was achieved with EOB-MRI.

In our study, several imaging modalities had a specificity of $100 \%$ for this purpose, which consisted of the presence of hypervascular foci within a nodule ("nodule-in-nodule" appearance) on HAP images of any imaging modalities, fat-containing appearance determined by hypoattenuation on unenhanced CT or a combination of in- and opposed-phase T1-weighted MR images (T1WI), and portal-flow decrease to any degree (hypoattenuation) on CTAP. However, the sensitivities of each imaging finding were low as evidenced by $10-20 \%$ for the "nodule-in-nodule" appearance obtained with HAP images, $42 \%$ and $52 \%$ for a fat-containing appearance obtained with unenhanced CT and a combination of T1WI, respectively, and $42 \%$ for a portal-flow decrease obtained with CTAP (table 1). 


\section{Liver Cancer}

\begin{tabular}{|c|c|}
\hline $\begin{array}{l}\text { DOI: } 10.1159 / 000343865 \\
\text { Published onlıne: IVlay 8, } 2014\end{array}$ & $\begin{array}{l}\text { (c) } 2014 \text { S. Karger AG, Basel } \\
\text { www.karger.com/lic }\end{array}$ \\
\hline
\end{tabular}
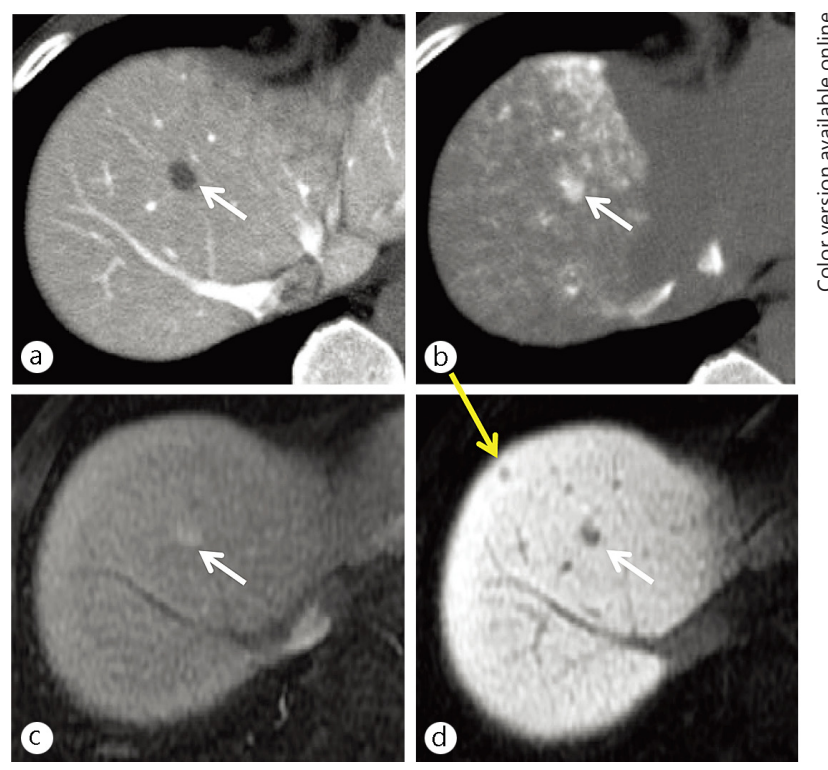

Fig. 3. A 58-year-old man with a conventional (hypervascular) HCC (white arrows) and an early HCC (yellow arrow). A small nodule was demonstrated as hypoattenuation (lack of portal flow) on CT imaging during arterioportography (a) and hyperattenuation (increased arterial flow) on CT imaging during hepatic arteriography (b). These findings are typical for a conventional (hypervascular) HCC. No additional nodule was not seen on these images. The conventional HCC showed hypervascularity on the hepatic arterial-dominant phase image (c) and hypointensity on the hepatocyte imaging (d) of the EOB-MRI. On hepatocyte imaging alone, a small hypointense nodule was demonstrated. The nodule was finally proven to be an early HCC based on surgicopathological examination.

Thus, we believe that the hypointensity observed on HP images of EOB-MRI made it the only imaging technique with high sensitivity and specificity for evaluating early HCC. Meanwhile, Bartolozzi et al. reported that 70\% (21/30) of high-grade DN showed hypointensity on HP images [16]. This discrepancy may be due to the different pathological criteria employed in each institution; there is a possibility that the nodules that were diagnosed as high-grade DN, by the previous pathological criteria, are now diagnosed as early HCC by the new ICGHN criteria. Following the ICGHN criteria becoming the standard, almost all surgically resected, early-stage hepatocellular nodules have been diagnosed as either a low-grade DN or an early HCC, and have never been categorized as a high-grade DN at our institution; this study [8] included no high-grade DN in contrast to the study by Bartolozzi et al. [16]. We must recognize that the pathological distinction between an early HCC and a high-grade DN may be extremely narrow and perhaps impossible in some instances, especially when diagnosing biopsy specimens [17]. Nevertheless, we emphasize that the finding of hypointensity on HP images of EOB-MRI is the most definite method to determine the diagnosis of an early HCC.

\section{Follow-Up Results for Hypovascular, Hepatocellular Nodules with EOB-MRI}

Hypointense Nodules on HP Images

As our investigation of the precise imaging-pathologic correlation has emphasized, almost all hypovascular, early-stage hepatocellular nodules showing hypointensity on HP images are an early HCC, and rarely a DN [8]. However, there may be some limitations, including the small sample size and the selection bias in this study. Generally, the majority of patients with such nodules have been followed-up and have rarely treated or even biopsied before the 
Table 1. Image findings with $100 \%$ specificity to distinguish early HCC from DN

\begin{tabular}{lll}
\hline Image findings & Modality & $\begin{array}{l}\text { Sensitivity } \\
\text { for early HCC }\end{array}$ \\
\hline $\begin{array}{l}\text { Hypervascularization } \\
\text { within nodule } \\
\text { (nodule-in-nodule appearance) }\end{array}$ & CTHA & $6 / 31(20 \%)$ \\
& HAP of CE-CT & $3 / 31(10 \%)$ \\
\hline $\begin{array}{l}\text { Fat-containing appearance } \\
\text { within nodule }\end{array}$ & HAP of EOB-MRI & $4 / 31(13 \%)$ \\
\hline $\begin{array}{l}\text { Decreased portal flow } \\
\text { within nodule } \\
\text { (to any degree) }\end{array}$ & $\begin{array}{l}\text { Combination of in- and } \\
\text { opposed-phase T1WI }\end{array}$ & $13 / 31(42 \%)$ \\
\hline $\begin{array}{l}\text { Hypointensity of nodule } \\
\text { CTAP }\end{array}$ & $16 / 31(52 \%)$ \\
\hline
\end{tabular}

nodules appeared to be hypervascular on HAP images, the sign which can convince clinicians that the patient should be diagnosed with a HCC. Therefore, it is important to clarify the fate of such nodules during the follow-up period before concluding that the majority of them are early HCC.

Several follow-up results for hypointense nodules on HP images have been recently reported, although it might be difficult to compare these reports because of varying follow-up periods and nodule sizes [18-21]. In our investigation, the 1-year cumulative risk of hypervascularization of such nodules was $15.6 \%$, but it increased to $37.6 \%$ for nodules greater than $10 \mathrm{~mm}$ in diameter [18] (fig. 4). Surprisingly, Kumada et al. reported that the 1-year cumulative risk of hypervascularization was $77.3 \%$ for nodules equal to or greater than $15 \mathrm{~mm}$ in diameter, although it was only $16.9 \%$ for nodules less than $15 \mathrm{~mm}$ in diameter [19], respectively. Similarly, Kim et al. and Hyodo et al. reported that $31-35 \%$ of such nodules (including those less than $5 \mathrm{~mm}$ in diameter) show hypervascularization during the follow-up period (mean, approximately 1 year) [20,21]. Considering that nodules showing hypointensity on HP images tend to appear hypervascular during the relatively short follow-up period, the majority of them might be treated as early HCC.

Hyperintense Nodules on HP Images

In contrast to the hypointense hepatocellular nodules, almost all hypovascular, hyperintense nodules seen on HP images are benign, including regenerative nodules or low-grade DN. Therefore, the implementation of follow-up studies for such hyperintense nodules seen on HP images might not be an issue that likely receives attention by most practicing hepatologists or radiologists.

However, some investigators have suggested the existence of early HCC showing isointensity or hyperintensity on HP images. Sano et al. reported that one of 31 early HCC cases $(1 / 31,3 \%)$ showed isointensity [8]. Chen et al. have reported three cases of early HCC showing hyperintensity on HP images [22]. In both reports, the diagnosis of early HCC for the nodules may be conclusive because of the detailed surgicopathological evaluation performed by pathological authorities (ICGHN members). Thus, radiologists and hepatologists should recognize that early HCC can show iso- or hyperintensity on HP images, similar to benign 

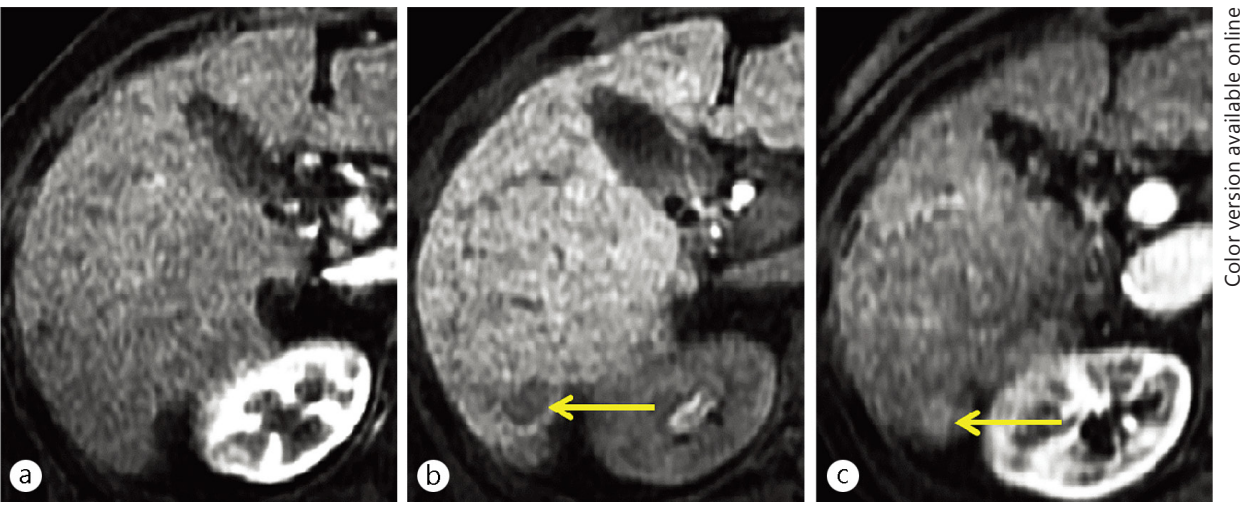

Fig. 4. An 81-year-old woman with a hypovascular, hypointense nodule on hepatocyte imaging of EOB-MRI (yellow arrows). A nodule of 12-mm in size was demonstrated showing hypovascularity (lack of arterial flow) on hepatic arterial-dominant phase imaging (a) and hypointensity on hepatocyte imaging (b) on the initial EOB-MRI examination. These findings are typical for an early (hypovascular) HCC. On follow-up hepatic arterial-dominant phase imaging of EOB-MRI (c), obtained three years after the initial EOB-MRI examination, early contrast-enhancement appeared within the nodule.

hepatocellular nodules, thereby making HCC a kind of "wolf in sheep's clothing" in such cases. Therefore, it is essential to know the optimal evidence-based management of these patients.

In our experience (unpublished data), the cumulative risk of hypervascularization is less than $0.5 \%$ per person-year (fig. 5). The cumulative risk of hypervascularization is significantly higher in nodules equal to or greater than $10 \mathrm{~mm}$ in diameter (1.3\% per person-year) than in those less than $10 \mathrm{~mm}$ in size ( $0.1 \%$ per person-year). The median time to hypervascularization is approximately two years. Our results suggest that the risk of hypervascularization for such nodules is extremely low, considering the incidence of HCC in patients with cirrhosis of the liver has been reported as approximately 8\% per 1-year [23] and 13\% per 3-years [24], respectively. The American Association for the Study of Liver Diseases (AASLD) practice guidelines for the management of HCC recommends ultrasound screening of patients with liver cirrhosis every 6 months [25]. Therefore, we believe that the low relevance of hyperintense nodules with a risk of hypervascularization detected on HP images of EOB-MRI, may not affect the AASLD recommendations for the clinical management of cirrhotic patients with such nodules.

\section{Current Consensus on the Diagnosis of Early HCC with EOB-MRI}

Based on several previous studies of imaging-pathologic correlation and the clinical follow-up for nodules showing hypointensity on HP images, it may be a worldwide consensus that almost all hypovascular, early-stage hepatocellular nodules showing hypointensity on HP images consist of a combination of a high proportion of early-HCC and a lower proportion of high-grade DN; these should be assumed to be a malignancy and treated accordingly. Dr. Kudo, who is a world renowned hepatologist and an Eastern opinion leader in this field, has stated that to assume hepatocellular nodules showing hypointensity on HP images are pathological early HCC cannot hurt in the clinical setting, and that such nodules may already have the indication for treatment [26]. Similarly, a review paper from Western countries has emphasized that EOB-MRI appears to be useful for the differentiation of an early HCC from a DN because 

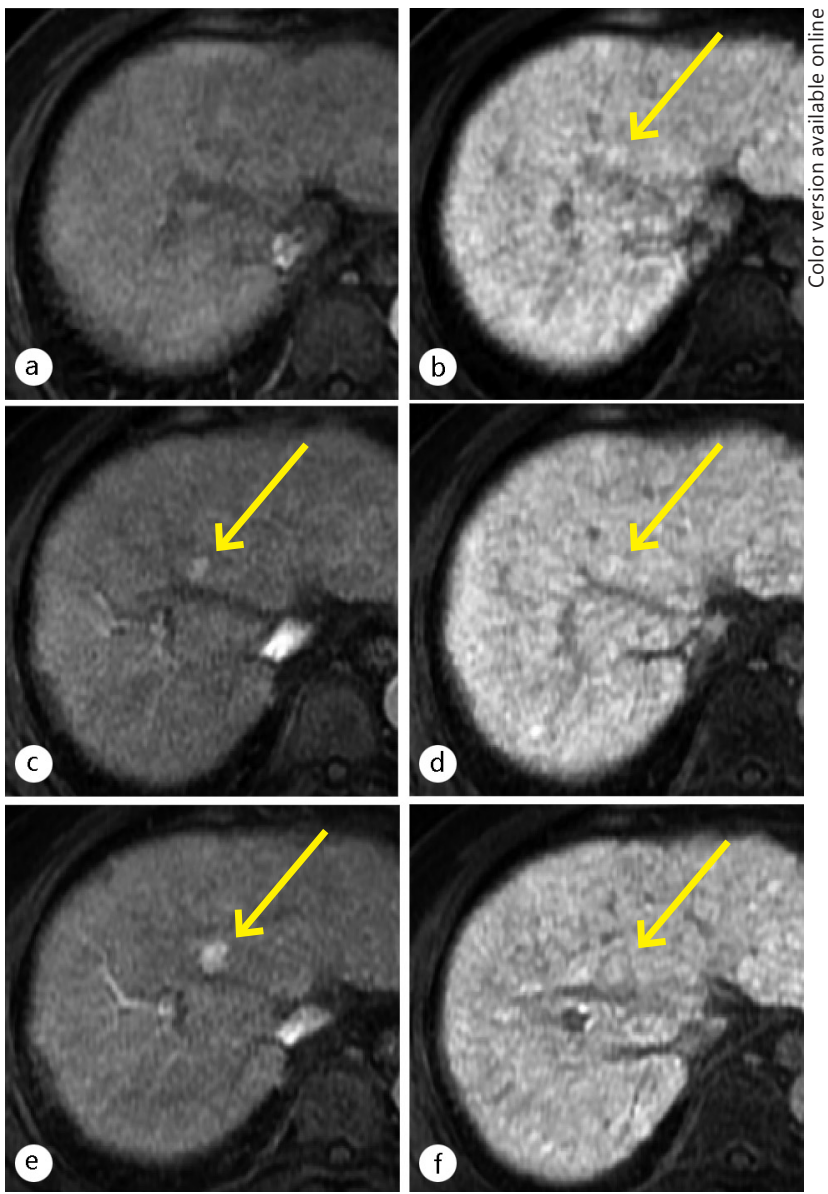

Fig. 5. A 57-year-old man with a hypovascular, hyperintense nodule on hepatocyte imaging of EOB-MRI (yellow arrows) (a, b). A nodule of 8-mm in size was demonstrated as slight hyperintensity on hepatocyte imaging (b) of the initial EOB-MRI examination. At this time, the nodule was not identified on the hepatic arterial-dominant phase imaging (a) because it did not show hypervascularity. On follow-up images of an EOB-MRI obtained 12 months after the initial examination, the nodule showed hypervascularity on hepatic arterial-dominant phase imaging (c). The nodule remained slightly hyperintense on hepatocyte imaging (d). On follow-up images of EOB-MRI obtained 17 months after the initial examination, the nodule with hypervascularity increased in size on hepatic arterial-dominant phase imaging (e) and hepatocyte imaging (f) . The nodule showed no interval change of the high signal intensity on hepatocyte imaging (f).

most HCC, including early HCC, appear hypointense as opposed to DN which do not usually show such hypointensity on HP images [27].

\section{Relationship between Signal Pattern of Nodules on HP Images and Expres- sion of OATP $1 B 3$ within Nodules}

It has been suggested that signal-intensity patterns on HP images of hypervascular, progressed HCC depend on the expression of OATP1B3 [28, 29] and may be related to the expression pattern of multidrug resistance protein (MRP) 2 within the nodules [30]. However, it is still unknown whether this theory can be also extended to the evaluation of early HCC, 


\section{Liver Cancer}
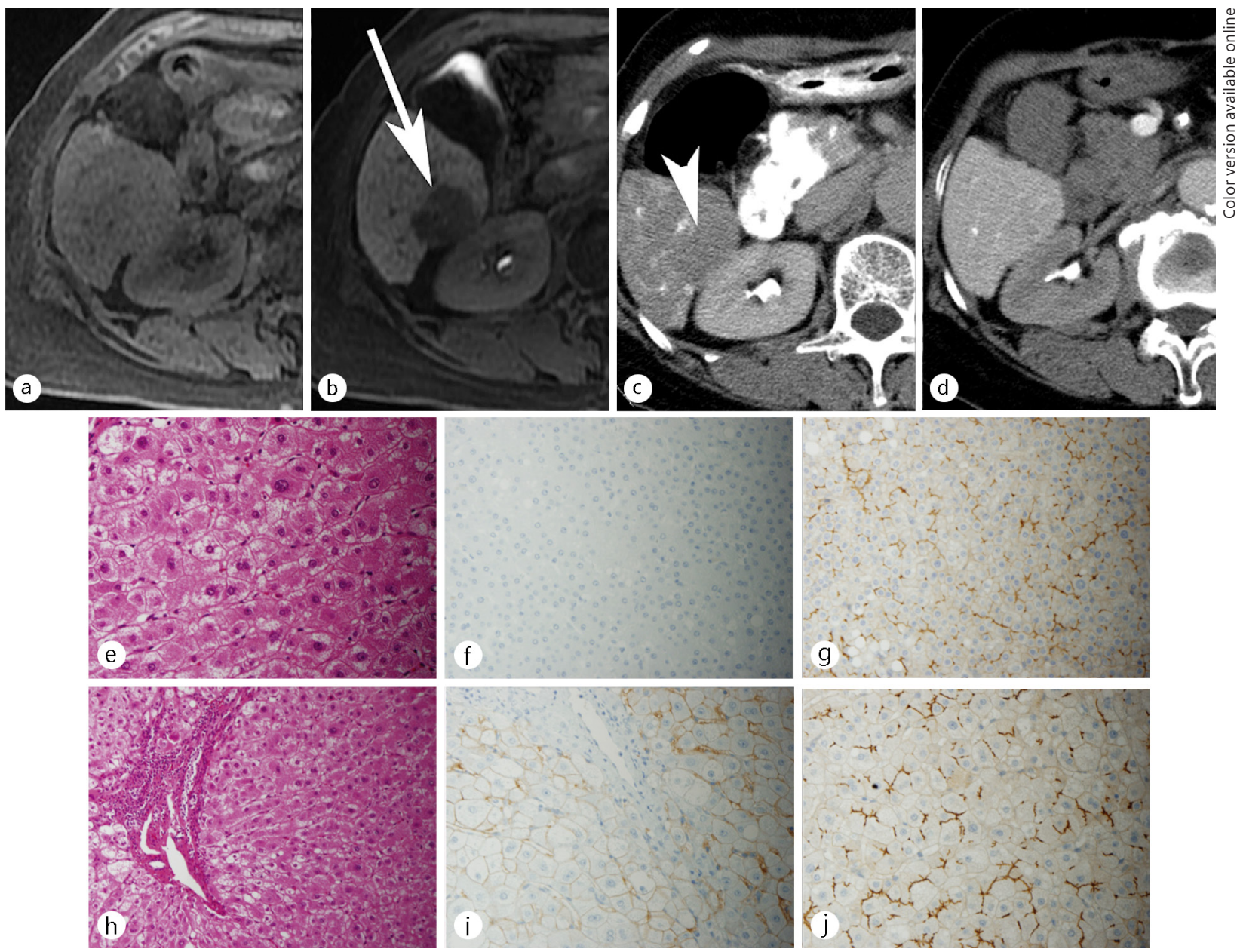

Fig. 6. A 58-year-old man with an early HCC. No lesion was shown on the unenhanced T1WI (a). Hepatocyte phase imaging (b) showed a well-defined nodule at the posterior segment of the right lobe of the liver (arrow). The nodule showed decreased uptake of gadexetic acid (relative enhancement ratio of 0.52 ), resulting in hypointensity relative to the surrounding liver. This nodule showed a slight decrease of arterial flow on CT imaging during hepatic arteriography (c), and equivalent of portal venous flow during arterioportography (d) compared to the surrounding liver. The histological examination of the nodule (e-g) compared with the surrounding liver (h-j), showed slight cellular atypia and mild increased cell density with thick trabecular pattern on hematoxylin and eosin stain (e), no expression of OATP1B3 (f), and equivalent expression of MRP2 (g) compared with surrounding liver (h, i, j, respectively).

as there has been a lack of data directly comparing the signal intensity on HP images with OATP1B3 expression in early HCC.

We have observed a good correlation between the signal-intensity patterns on HP images and OATP1B3 expression, even for early HCC and low-grade DN; more than $90 \%$ of the early HCC cases showing a decreased uptake of gadoxetic acid (hypointensity) on HP images showed no or decreased expression of OATP1B3 (fig. 6). In addition, 100\% of the early HCC and low-grade DN cases showing increased uptake of gadoxetic acid (hyperintensity) on HP images showed increased expression of OATP1B3. Furthermore, there was no correlation between the signal-intensity patterns on HP images of the early-stage nodules and MRP2 expression.

Our results may indicate that the excellent, highly-objective diagnostic ability of HP images of EOB-MRI for diagnosing early HCC can be realized pathologically by introducing the 
OATP1B3 staining method. For example, the OATP1B3 staining method may be helpful for the diagnosis of very small biopsy specimens in which "stromal invasion," which is the most important ICGHN criterion for diagnosing early HCC, might not be seen. If absent or decreased expression of OATP1B3 can be identified within such biopsy specimens, early HCC can be diagnosed from such nodules.

\section{Conclusion}

There is no doubt that EOB-MRI has achieved a major breakthrough for the detection of early HCC and the differentiation between an early HCC and a DN. In particular, a hypointense appearance seen on HP images is the most important imaging finding for such purposes. Using this imaging finding, both the sensitivity and specificity in the diagnosis of early HCC are almost perfect. At this time, it is just beginning to be accepted worldwide that almost all hypovascular, early-stage hepatocellular nodules showing hypointensity on HP images are early HCC, although a discrepancy in the pathological opinion remains in different institutions.

Recently, it has been elucidated that the signal-intensity patterns on HP images of EOBMRI of early-stage hepatocellular nodules, as well as progressed HCC, are directly relevant to the expression of OATP1B3; that is, hypointense nodules seen on HP images have absent or decreased expression of OATP1B3, and that the opposite is equally true. Therefore, the diagnostic utility of pathology for the diagnosis of early HCC may be dramatically improved by introducing the OATP1B3 staining method.

\section{References}

1 Kudo M: Early hepatocellular carcinoma: definition and diagnosis. Liver Cancer 2013;2:69-72.

-2 Kudo M: Multistep human hepatocarcinogenesis: correlation of imaging with pathology. J Gastroenterol 2009;44(Suppl 19):112-118.

-3 Kitao A, Zen Y, Matsui O, Gabata T, Nakanuma Y: Hepatocarcinogenesis: multistep changes of drainage vessels at CT during arterial portography and hepatic arteriography-radiologic-pathologic correlation. Radiology 2009;252:605-614.

4 Sakamoto M, Hirohashi S, Shimosato Y: Early stages of multistep hepatocarcinogenesis: adenomatous hyperplasia and early hepatocellular carcinoma. Hum Pathol 1991;22:172-178.

5 International Working Party: Terminology of nodular hepatocellular lesions. Hepatology 1995;22:983993.

6 Desmet VJ: East-West pathology agreement on precancerous liver lesions and early hepatocellular carcinoma. Hepatology 2009;49:355-357.

-7 The International Consensus Group for Hepatocellular Neoplasia: Pathologic diagnosis of early hepatocellular carcinoma: a report of the international consensus group for hepatocellular neoplasia. Hepatology 2009;49:658-664.

-8 Sano K, Ichikawa T, Motosugi U, Sou H, Muhi AM, Matsuda M, Nakano M, Sakamoto M, Nakazawa T, Asakawa M, Fujii H, Kitamura T, Enomoto N, Araki T: Imaging study of early hepatocellular carcinoma: usefulness of gadoxetic acid-enhanced MR imaging. Radiology 2011;261:834-844.

9 Lee JM, Yoon JH, Joo I, Woo HS: Recent advances in CT and MR imaging for evaluation of hepatocellular carcinoma. Liver Cancer 2012;1:22-40.

10 Vogl TJ, Kümmel S, Hammerstingl R, Schellenbeck M, Schumacher G, Balzer T, Schwarz W, Müller PK, Bechstein WO, Mack MG, Söllner O, Felix R: Liver tumors: comparison of MR imaging with Gd-EOB-DTPA and Gd-DTPA. Radiology 1996;200:59-67.

11 Reimer P, Rummeny EJ, Shamsi K, Balzer T, Daldrup HE, Tombach B, Hesse T, Berns T, Peters PE: Phase II clinical evaluation of Gd-EOB-DTPA: dose, safety aspects, and pulse sequence. Radiology 1996;199:177183.

12 Huppertz A, Haraida S, Kraus A, Zech CJ, Scheidler J, Breuer J, Helmberger TK, Reiser MF: Enhancement of focal liver lesions at gadoxetic acid-enhanced MR imaging: correlation with histopathologic findings and spiral CT—initial observations. Radiology 2005;234:468-478. 
13 Hammerstingl R, Huppertz A, Breuer J, Balzer T, Blakeborough A, Carter R, Fusté LC, Heinz-Peer G, Judmaier W, Laniado M, Manfredi RM, Mathieu DG, Müller D, Mortelè K, Reimer P, Reiser MF, Robinson PJ, Shamsi K, Strotzer M, Taupitz M, Tombach B, Valeri G, van Beers BE, Vogl TJ, European EOB-study group: Diagnostic efficacy of gadoxetic acid (Primovist)-enhanced MRI and spiral CT for a therapeutic strategy: comparison with intraoperative and histopathologic findings in focal liver lesions. Eur Radiol 2008;18:457-467.

-14 Zech CJ, Grazioli L, Breuer J, Reiser MF, Schoenberg SO: Diagnostic performance and description of morphological features of focal nodular hyperplasia in Gd-EOB-DTPA-enhanced liver magnetic resonance imaging: results of a multicenter trial. Invest Radiol 2008;43:504-511.

15 Ichikawa T, Saito K, Yoshioka N, Tanimoto A, Gokan T, Takehara Y, Kamura T, Gabata T, Murakami T, Ito K, Hirohashi S, Nishie A, Saito Y, Onaya H, Kuwatsuru R, Morimoto A, Ueda K, Kurauchi M, Breuer J: Detection and characterization of focal liver lesions: a Japanese phase III, multicenter comparison between gadoxetic acid disodium-enhanced magnetic resonance imaging and contrast-enhanced computed tomography predominantly in patients with hepatocellular carcinoma and chronic liver disease. Invest Radiol 2010;45:133-141.

16 Bartolozzi C, Battaglia V, Bargellini I, Bozzi E, Campani D, Pollina LE, Filipponi F: Contrast-enhanced magnetic resonance imaging of 102 nodules in cirrhosis: correlation with histological findings on explanted livers. Abdom Imaging 2013;38:290-296.

17 Kojiro M: Diagnostic discrepancy of early hepatocellular carcinoma between Japan and West. Hepatol Res 2007;37(Suppl 2):S121-S124.

18 Motosugi U, Ichikawa T, Sano K, Sou H, Onohara K, Muhi A, Amemiya F, Enomoto N, Matsuda M, Fujii H, Araki T: Outcome of hypovascular hepatic nodules revealing no gadoxetic acid uptake in patients with chronic liver disease. J Magn Reson Imaging 2011;34:88-94.

19 Kumada T, Toyoda H, Tada T, Sone Y, Fujimori M, Ogawa S, Ishikawa T: Evolution of hypointense hepatocellular nodules observed only in the hepatobiliary phase of gadoxetate disodium-enhanced MRI. AJR Am J Roentgenol 2011;197:58-63.

20 Kim YK, Lee WJ, Park MJ, Kim SH, Rhim H, Choi D: Hypovascular hypointense nodules on hepatobiliary phase gadoxetic acid-enhanced MR images in patients with cirrhosis: potential of DW imaging in predicting progression to hypervascular HCC. Radiology 2012;265:104-114.

-21 Hyodo T, Murakami T, Imai Y, Okada M, Hori M, Kagawa Y, Kogita S, Kumano S, Kudo M, Mochizuki T: Hypovascular nodules in patients with chronic liver disease: risk factors for development of hypervascular hepatocellular carcinoma. Radiology 2013;266:480-490.

22 Chen N, Motosugi U, Sano K, Ichikawa T, Nakano M, Morisaka H, Ichikawa S, Matsuda M, Fujii H, Enomoto $\mathrm{N}$, Araki T: Early hepatocellular carcinomas showing isointensity or hyperintensity in gadoxetic acid-enhanced, hepatocyte-phase magnetic resonance images. J Comput Assist Tomogr 2013;37:466-469.

-23 Yoshida H, Shiratori Y, Moriyama M, Arakawa Y, Ide T, Sata M, Inoue O, Yano M, Tanaka M, Fujiyama S, Nishiguchi S, Kuroki T, Imazeki F, Yokosuka O, Kinoyama S, Yamada G, Omata M: Interferon therapy reduces the risk for hepatocellular carcinoma: national surveillance program of cirrhotic and noncirrhotic patients with chronic hepatitis C in Japan. IHIT Study Group. Inhibition of Hepatocarcinogenesis by Interferon Therapy. Ann Intern Med 1999;131:174-181.

-24 Tsukuma H, Hiyama T, Tanaka S, Nakao M, Yabuuchi T, Kitamura T, Nakanishi K, Fujimoto I, Inoue A, Yamazaki $\mathrm{H}$, et al: Risk factors for hepatocellular carcinoma among patients with chronic liver disease. $\mathrm{N}$ Engl J Med 1993;328:1797-1801.

25 Bruix J, Sherman M American Association for the Study of Liver Diseases: Management of hepatocellular carcinoma: an update. Hepatology 2011;53:1020-1022.

26 Kudo M: Natural course of hypovascular nodule, which shows low intense on hepatobiliary phase Gd-EOBDTPA. Kantansuigazo 2012;14:369-370.

27 Van Beers BE, Pastor CM, Hussain HK: Primovist, Eovist: what to expect? J Hepatol 2012;57:421-429.

28 Kitao A, Zen Y, Matsui O, Gabata T, Kobayashi S, Koda W, Kozaka K, Yoneda N, Yamashita T, Kaneko S, Nakanuma Y: Hepatocellular carcinoma: signal intensity at gadoxetic acid-enhanced MR Imaging-correlation with molecular transporters and histopathologic features. Radiology 2010;256:817-826.

29 Narita M, Hatano E, Arizono S, Miyagawa-Hayashino A, Isoda H, Kitamura K, Taura K, Yasuchika K, Nitta T, Ikai I, Uemoto S: Expression of OATP1B3 determines uptake of Gd-EOB-DTPA in hepatocellular carcinoma. J Gastroenterol 2009;44:793-798.

-30 Tsuboyama T, Onishi H, Kim T, Akita H, Hori M, Tatsumi M, Nakamoto A, Nagano H, Matsuura N, Wakasa K, Tomoda K: Hepatocellular carcinoma: hepatocyte-selective enhancement at gadoxetic acid-enhanced MR imaging-correlation with expression of sinusoidal and canalicular transporters and bile accumulation. Radiology 2010;255:824-833. 